\title{
Comparison of the Prevalence of Low Back Pain and Related Spinal Diseases among Smokers and Nonsmokers: Using Korean National Health Insurance Database
}

\author{
Ji-Won Kwon, MD ${ }^{*, \dagger}$, Joong-Won Ha, MD ${ }^{*, \dagger}$, Tae-Sung Lee, $\mathrm{MD}^{*}$, \\ Seong-Hwan Moon, $\mathrm{MD}^{*}$, Hwan-Mo Lee, $\mathrm{MD}^{*}$, Yung Park, $\mathrm{MD}^{*, \dagger}$ \\ ${ }^{*}$ Department of Orthopedic Surgery, Yonsei University College of Medicine, Seoul, \\ ${ }^{\dagger}$ Department of Orthopedic Surgery, National Health Insurance Service Ilsan Hospital, Goyang, Korea
}

Background: To compare the risk of low back pain (LBP) and related spinal diseases between smokers (exposure group) and nonsmokers (non-exposure group). No large registry study has so far investigated the association between smoking and LBP-related spinal diseases such as intervertebral disc disease, spinal stenosis, spinal instability, and spondylolisthesis.

Methods: A random sample was taken from the Korean National Health Insurance Research Database. In total, 204,066 men $(160,105$ smokers, 43,961 nonsmokers) who were followed up between 2002 and 2013 were included in the analysis. Patients with previous back pain or spinal disease in 2003 and 2004, patients with inappropriate data, and women (due to the lower percentage of smokers) were excluded. The Cox proportional hazard model was used to investigate the risk of LBP and related spinal diseases associated with smoking, while adjusting for demographic, clinical, and socioeconomic factors.

Results: The overall median follow-up period was 5.6 years (interquartile range, $3.48-8.43$ years). Compared to the nonsmoker group, the smoker group had a higher incidence of LBP $(p=0.01)$, intervertebral disc disease $(p<0.001)$, spinal stenosis $(p=0.004)$, spinal instability $(p<0.001)$, and spondylolisthesis $(p=0.023)$. Compared to the nonsmoker group, the smoker group had a higher adjusted hazard ratio (HR) per year of LBP (HR, 1.18; 95\% confidence interval [Cl], 1.15 to 1.21), intervertebral disc disease (HR, 1.25; 95\% Cl, 1.21 to 1.30), spinal stenosis ( $\mathrm{HR}, 1.52 ; 95 \% \mathrm{Cl}, 1.41$ to 1.64), spinal instability ( $\mathrm{HR}, 1.33 ; 95 \% \mathrm{Cl}, 1.24$ to 1.44), and spondylolisthesis (HR, 1.49; 95\% Cl, 1.23 to 1.80).

Conclusions: Smokers in male samples were at higher risk for LBP and related spinal diseases than nonsmokers.

Keywords: Smoker, Low back pain, Spinal disease, Big data, Prevalence

Low back pain (LBP) is a highly prevalent and burdensome health problem worldwide and the leading cause of disability in most countries. ${ }^{1,2)}$ Its high recurrence rate within 1 year after recovery from a previous episode is a

Received June 27, 2019; Accepted August 22, 2019

Correspondence to: Yung Park, MD

Department of Orthopedic Surgery, National Health Insurance Service

Ilsan Hospital, 100 Ilsan-ro, Ilsandong-gu, Goyang 10444, Korea

Tel: +82-31-900-0270, Fax: +82-31-900-0343

E-mail: yungspine@gmail.com key contributor to the recognition of LBP as the public health burden. ${ }^{3-5)}$ Smoking has been suggested as a risk factor for LBP. ${ }^{6,7)}$ However, evidence on the role of smoking in the development of LBP is inconsistent, ${ }^{8-10)}$ and the exact nature of that link has been largely untested in longterm cohort studies.

In a recent meta-analysis ${ }^{10)}$ of 40 studies examining the relationship between smoking and LBP, current smokers had a $31 \%$ higher risk of LBP than nonsmokers, but that estimate applied only the preceding 12 months. None of the included studies were designed to determine wheth-

Copyright (C) 2020 by The Korean Orthopaedic Association 
Kwon et al. Prevalence of Spinal Disease among Smokers in Korea

Clinics in Orthopedic Surgery • Vol. 12, No. 2, $2020 \bullet$ www.ecios.org

er there is a cause and effect relationship, which cannot be established in a randomized controlled trial due to ethical concerns. Therefore, the most feasible study design for assessing the relationship in humans is a cohort study.

Another systematic review demonstrated that the data were fairly consistent with the notion that smoking is associated with LBP, but there were too few studies to make any conclusions regarding other conditions, such as disc degeneration, herniated discs, and sciatica. ${ }^{8)}$ Furthermore, no large registry study has so far investigated the association between smoking and LBP-related lumbar spine diseases, such as intervertebral disc disease, spinal stenosis, spinal instability, or spondylolisthesis. We, therefore, reviewed a random sample taken from the Korean National Health Insurance Research Database with the aim of investigating whether smoking can be a potential risk for LBP and related spinal diseases and, in particular, whether the risk is higher in the smokers (exposure group) than nonsmokers (non-exposure group).

\section{METHODS}

The Korean National Health Insurance Service (NHIS) has provided a national health screening program since 1995. The NHIS constructed a sample cohort database in 2015 to offer relevant, useful data for health research, especially in the field of noncommunicable diseases and health risk factors. For this study, we used this database, which is registered as the NHIS-Health Screening Cohort. We conducted this study in compliance with the principles of the Declaration of Helsinki. The protocol of this study was reviewed and approved by the Institutional Review Board and Ethics Committee of National Health Insurance Service Ilsan Hospital (IRB No. 2015-02-037). The informed consent was waived.

\section{Study Population}

The cohort was first selected from the 2002 and 2003 health screening participants, who were aged between 40 years and 79 years in 2002 and followed up through 2013. This initial cohort included 514,866 health screening participants who comprised $10 \%$ of a simple random sample of all national health screening participants in 2002 and 2003. The database meets the high demand for research requiring both health screening data and long-term health outcomes including detailed information on medical treatment, health screening, and socioeconomic variables (residence, death date, cause of death, and income level). The complete profile of the cohort has been published recently. ${ }^{11)}$

To minimize the possibility of selection bias, we excluded 181,766 patients (74,466 men and 107,300 women) diagnosed as having back pain or spinal diseases during the first 2 years (2002-2003). We also excluded 911 participants (593 men and 318 women) whose baseline smoking status was unknown. The proportion of women who reported themselves as smokers was too small $(7,204,5.6 \%)$, compared to that of male participants, to produce statistically relevant results. Therefore, we excluded all women from analysis. Finally, the investigated cohort consisted of 204,066 men (160,105 smokers and 43,961 nonsmokers) (Fig. 1).

\section{Data Collection}

Health screening participants undergo standardized examinations per 2 years. During visits, they report their smoking habits, medical history, alcohol consumption, and physical activity. Weight and height are measured in light clothing. Blood pressure is measured in a seated position. Blood samples are obtained after an overnight fast for white cell count and clinical chemical analysis. Quality

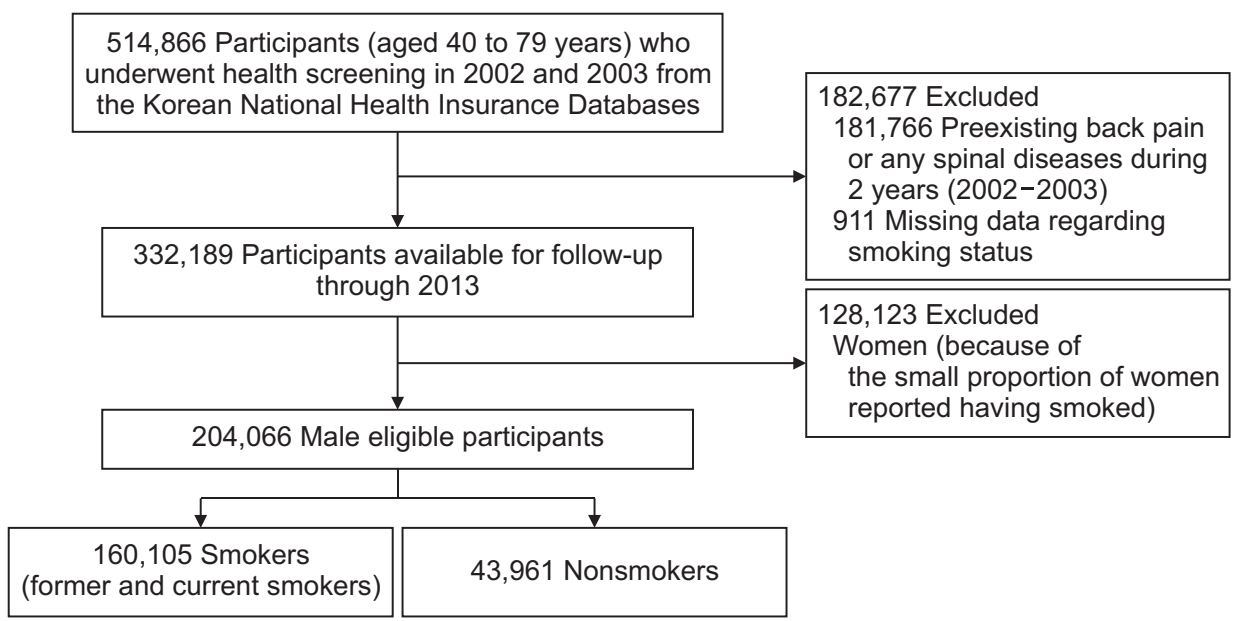

Fig. 1. Flowchart of study population selection. 
Kwon et al. Prevalence of Spinal Disease among Smokers in Korea

Clinics in Orthopedic Surgery • Vol. 12, No. 2, $2020 \bullet$ www.ecios.org

control procedures are performed in accordance with the guidelines of the Korean Association of Laboratory Quality Control.

Information on smoking was obtained from all participants at least once between 2002 and 2013. All smoking data were self-reported. The health examination questionnaire contains questions about smoking history, the number of cigarettes consumed per day, years of smoking duration, and years since cessation. The responses in serial follow-up questionnaires were used to model smoking as a time-dependent variable. Former smokers and participants who had never smoked did not complete follow-up questionnaires reflecting their smoking history (we did not censor these data). We censored the data of current smokers on the date of the second missed questionnaire to prevent misclassifying those who might have quit smoking.

\section{Ascertainment of LBP and Other Outcomes}

The primary outcome was LBP and four common lumbar or lumbosacral spinal diseases-intervertebral disc disease, spinal stenosis, spinal instability, and spondylolisthesis. All diagnoses of LBP and the four spinal diseases described in the International Classification of Diseases and Related Health Problems, Tenth Revision (ICD-10) were included. We excluded back pain or spinal diseases attributed to inflammatory, infectious, traumatic, neoplastic, deformed, or congenital causes. The site of involvement was restricted to lumbar or lumbosacral by supplementary subclassifications, which were provided for optional use in the appropriate categories in the block on spondylopathy, except in categories M51 and M54.5. The final analysis included five outcomes: LBP (M54.5) and four common lumbar or lumbosacral spinal diseases-intervertebral disc disorders (M51), spinal stenosis (M4806, M4807), spinal instability (M5326, M5327), and spondylolisthesis (M4316, M4317). We tried to minimize the effect of previous back pain or any spinal diseases (M40-M54) on the baseline by excluding all events that occurred during the first two years of follow-up (2002 and 2003). Thus, general followup began on January 1 of the 3rd year after the baseline visit.

\section{Statistical Analysis}

To investigate differences between smoking exposure (smoker) and non-exposure (nonsmoker) groups, the Fisher exact test was used for categorical variables and the $t$-test for continuous variables if normally distributed; if not, the Mann-Whitney test was used. For a given covariate, a standardized difference of less than $10 \%$ indicates a rela- tively small imbalance. ${ }^{12)}$ Event rates per 10,000 personyears were calculated as number of events divided by total follow-up time until the event, death, or censoring per 10,000 years, with $95 \%$ confidence intervals (CIs) estimated by assuming Poisson distribution. The difference between two event rates was tested by assuming approximately normal distribution of the estimates with the $\mathrm{Z}$ test statistic. We calculated unadjusted rates of LBP and the four lumbar spinal diseases per person-year and produced Kaplan-Meier survival curves (survival and rms packages, R software, ver. 3.3.2, The R Foundation, Vienna, Austria) for smoking status.

To compare the risk of LBP and related lumbar spinal diseases between the groups, a Cox proportional hazards model starting at baseline was used. We modeled time to event. The Cox regression model used the length of each individual's follow-up period. Follow-up for all participants began at the time of the original health examination between 2002 and 2003 and was concluded on the date of development of any of the morbidities, which is the date on which data were censored, or December 31, 2013, whichever came first. Cox analyses were performed for LBP, intervertebral disc disease, spinal stenosis, spinal instability, and spondylolisthesis. In these analyses, we compared the morbidity risk in the smoker (former and current smokers) group with that in the nonsmoker group.

The multivariable Cox model was adjusted for the age at enrollment (continuous variable), body mass index (continuous variable), alcohol consumption (five categories based on frequency: never, 2-3 per month, 1-2 per week, 3-4 per week, and everyday), physical activity (five categories based on frequency: never, 1-2 per week, 3-4 per week, 5-6 per week, and everyday), socioeconomic status (three categories based on income: high, middle, and low), total cholesterol (continuous variable), and blood sugar (continuous variable). All analyses were accomplished using SAS ver. 9.4 (SAS Institute, Cary, NC, USA).

\section{RESULTS}

The standardized differences between the groups were below $10 \%$ for all investigated baseline characteristics except for the mean age, alcohol consumption, and physical activity (Table 1). The smoker group was slightly younger than the nonsmoker group with standardized difference of $24.7 \%$. More alcohol consumption and physical activity were reported in the smoker group than in the nonsmoker group, with a difference of $55.3 \%$ and $17.9 \%$, respectively. The overall median follow-up period of all 72,814 patients 
Kwon et al. Prevalence of Spinal Disease among Smokers in Korea

Clinics in Orthopedic Surgery • Vol. 12, No. 2, 2020 • www.ecios.org

Table 1. Baseline Demographic Characteristics of Male Participants According to Smoking Status

\begin{tabular}{|c|c|c|c|}
\hline Characteristic & Nonsmoker group $(n=43,961)$ & Smoker group $(n=160,105)$ & Standardized difference (\%) \\
\hline Pack-year & - & 18.59 (8.63-25.88) & \\
\hline Age (yr) & $56.53 \pm 9.99$ & $54.18 \pm 8.99$ & 24.7 \\
\hline Body mass index $\left(\mathrm{kg} / \mathrm{m}^{2}\right)$ & $24.15(22.27-25.95)$ & $23.95(22.05-225.77)$ & 7.0 \\
\hline \multicolumn{4}{|l|}{ Blood chemistry } \\
\hline Blood sugar & $100.32(84-105)$ & $101.08(85-2106)$ & 2.2 \\
\hline Total cholesterol & $196.69(172-219)$ & $198.10(173-2221)$ & 3.8 \\
\hline SGOT (AST) & $28.64(21-31)$ & $29.04(18-234)$ & 2.0 \\
\hline SGPT (ALT) & $29.49(18-34)$ & $29.82(18-234)$ & 1.4 \\
\hline \multicolumn{4}{|l|}{ Medical history* } \\
\hline Hypertension & $2,435(7.1)$ & $7,743(6.2)$ & 3.7 \\
\hline Heart disease & $332(1.0)$ & $1,153(0.9)$ & 0.5 \\
\hline Stroke & $180(0.5)$ & $428(0.3)$ & 2.8 \\
\hline Diabetes & $1,306(3.8)$ & $4,417(3.5)$ & 1.5 \\
\hline \multicolumn{4}{|l|}{ Alcohol consumption* } \\
\hline Nondrinker & $21,549(49.2)$ & $40,207(25.2)$ & 55.3 \\
\hline 2-3 Drinks per month & $8,089(18.5)$ & $30,504(19.1)$ & \\
\hline 1-2 Drinks per week & $9,085(20.7)$ & $51,839(32.5)$ & \\
\hline 3-4 Drinks per week & $3,204(7.3)$ & $23,769(14.9)$ & \\
\hline Everyday drinker & $1,907(4.4)$ & $13,390(8.4)$ & \\
\hline Physical activity (exercise frequency)* & & & 17.9 \\
\hline None & $19,933(46.3)$ & $64,469(40.4)$ & \\
\hline 1-2 Per week & $12,991(30.2)$ & $46,535(29.2)$ & \\
\hline 3-4 Per week & $4,989(11.6)$ & $20,483(12.8)$ & \\
\hline 5-6 Per week & $1,345(3.1)$ & $6,797(4.3)$ & \\
\hline Everyday & 3,774 (8.8) & $21,202(13.3)$ & \\
\hline Socioeconomic status $^{\dagger}$ & & & 6.9 \\
\hline High & $18,320(41.7)$ & $61,312(38.3)$ & \\
\hline Middle & $15,326(34.9)$ & $39,288(24.5)$ & \\
\hline Low & $10,315(23.5)$ & $59,505(37.2)$ & \\
\hline
\end{tabular}

Values are presented as mean \pm standard deviation, mean (interquartile range), or number (\%).

SGOT: serum glutamic oxaloacetic transaminase, AST: aspartate transaminase, SGPT: serum glutamic pyruvic transaminase, ALT: alanine transaminase. * Medical history, alcohol consumption, and physical activity were self-reported. 'Socioeconomic status was classified into three categories based on income.

who developed LBP or the four lumbar spinal diseases was 5.6 years (interquartile range [IQR], 3.48 to 8.43 years). The overall mean age at the time of the event was $56.6 \pm 8.9$ years. There were 44,937 patients with LBP, 20,194 patients with intervertebral disc disease, 4,412 patients with spinal stenosis, 4,585 patients with spinal instability, and 1,143 
Kwon et al. Prevalence of Spinal Disease among Smokers in Korea

Clinics in Orthopedic Surgery • Vol. 12, No. 2, 2020 • www.ecios.org

Table 2. Risk of Low Back Pain and Related Low Back Spinal Diseases According to Smoking Status in Men

\begin{tabular}{|c|c|c|c|}
\hline Variable & Nonsmoker group $(n=43,961)$ & Smoker group $(n=160,105)$ & $p$-value \\
\hline \multicolumn{4}{|l|}{ Low back pain (M545) } \\
\hline Median duration of follow-up (IOR), yr & $5.89(3.62-8.74)$ & & \\
\hline No. $(\%)$ & $9,654(21.96)$ & $35,283(22.04)$ & 0.01 \\
\hline Incidence rate per 10,000 person-year (95\% CI) & 473.08 (463.64-482.52) & $1729.00(1710.96-1747.04)$ & 0.01 \\
\hline Difference per 10,000 person-year (95\% CI) & Reference & 1255.92 (1255.76-1256.07) & $<0.001$ \\
\hline Multivariable-adjusted HR per year $(95 \% \mathrm{CI})^{*}$ & 1 (Reference) & $1.18(1.15-1.21)$ & $<0.001$ \\
\hline \multicolumn{4}{|l|}{ Intervertebral disc disease (M51) } \\
\hline Median duration of follow-up (IOR), yr & $4.79(3.14-7.40)$ & & \\
\hline No. $(\%)$ & $4,180(9.51)$ & $16,014(10.00)$ & $<0.001$ \\
\hline Incidence rate per 10,000 person-year (95\% CI) & 204.84 (198.63-211.05) & 784.75 (772.60-796.90) & $<0.001$ \\
\hline Difference per 10,000 person-year (95\% CI) & Reference & $579.91(579.81-580.01)$ & $<0.001$ \\
\hline Multivariable-adjusted HR per year $(95 \% \mathrm{CI})^{*}$ & 1 (Reference) & $1.25(1.21-1.30)$ & $<0.001$ \\
\hline \multicolumn{4}{|l|}{ Spinal stenosis (M4806, M4807) } \\
\hline Median duration of follow-up (IQR), yr & $7.64(5.50-9.73)$ & & \\
\hline No. $(\%)$ & $931(2.12)$ & $3,481(2.17)$ & 0.004 \\
\hline Incidence rate per 10,000 person-year (95\% CI) & 45.62 (42.69-48.55) & $170.58(164.91-176.25)$ & 0.004 \\
\hline Difference per 10,000 person-year (95\% CI) & Reference & $124.96(124.91-125.01)$ & $<0.001$ \\
\hline Multivariable-adjusted HR per year $(95 \% \mathrm{CI})^{*}$ & 1 (Reference) & $1.52(1.41-1.64)$ & $<0.001$ \\
\hline \multicolumn{4}{|l|}{ Spinal instability (M5326, M5327) } \\
\hline Median duration of follow-up (IOR), yr & $4.94(3.26-7.58)$ & & \\
\hline No. $(\%)$ & $915(2.08)$ & $3,670(2.29)$ & $<0.001$ \\
\hline Incidence rate per 10,000 person-year (95\% CI) & $44.84(41.93-47.75)$ & $179.84(174.02-185.66)$ & $<0.001$ \\
\hline Difference per 10,000 person-year (95\% CI) & Reference & $135.01(134.95-135.06)$ & $<0.001$ \\
\hline Multivariable-adjusted HR per year $(95 \% \mathrm{CI})^{*}$ & 1 (Reference) & $1.33(1.24-1.44)$ & $<0.001$ \\
\hline \multicolumn{4}{|l|}{ Spondylolisthesis (M4316, M4317) } \\
\hline Median duration of follow-up (IQR), yr & $6.66(4.94-8.71)$ & & \\
\hline No. $(\%)$ & $243(0.55)$ & $900(0.56)$ & 0.023 \\
\hline Incidence rate per 10,000 person-year (95\% CI) & $11.91(10.41-13.41)$ & 44.10 (41.22-46.98) & 0.023 \\
\hline Difference per 10,000 person-year (95\% CI) & Reference & $32.20(32.17-32.22)$ & $<0.001$ \\
\hline Multivariable-adjusted HR per year $(95 \% \mathrm{CI})^{*}$ & 1 (Reference) & $1.49(1.23-1.80)$ & $<0.001$ \\
\hline
\end{tabular}

IOR: interquartile range, Cl: confidence interval, HR: hazard ratio.

*HRs were estimated from Cox proportional hazards models adjusted for age, body mass index, total cholesterol, blood sugar, alcohol consumption (five categories based on frequency: never, 2-3 per month, 1-2 per week, 3-4 per week, and everyday), physical activity (five categories based on frequency: never, 1-2 per week, 3-4 per week, 5-6 per week, and everyday), and socioeconomic status (three categories based on income: high, middle, and low). 
Kwon et al. Prevalence of Spinal Disease among Smokers in Korea

Clinics in Orthopedic Surgery • Vol. 12, No. 2, $2020 \bullet$ www.ecios.org

patients with spondylolisthesis. After a median follow-up of 5.9 years (IQR, 3.6 to 8.7 years), there were 35,283 cases of LBP in the smoker group and 9,654 cases of LBP in the nonsmoker group, corresponding to incidence rates of $1,729.00$ (95\% CI, $1,710.96$ to $1,747.04)$ and 473.08 (95\% CI, 463.64 to 482.52 ) cases of LBP per 10,000 person- years, with an absolute rate difference of 1,255.92 (95\% CI, $1,255.76$ to $1,256.07)$. The incidence of each of the four spinal diseases (intervertebral disc disease, spinal stenosis, spinal instability, and spondylolisthesis) was also higher in the smoker group, with a higher incidence rate per 10,000 person-years and an absolute rate difference (Table 2).

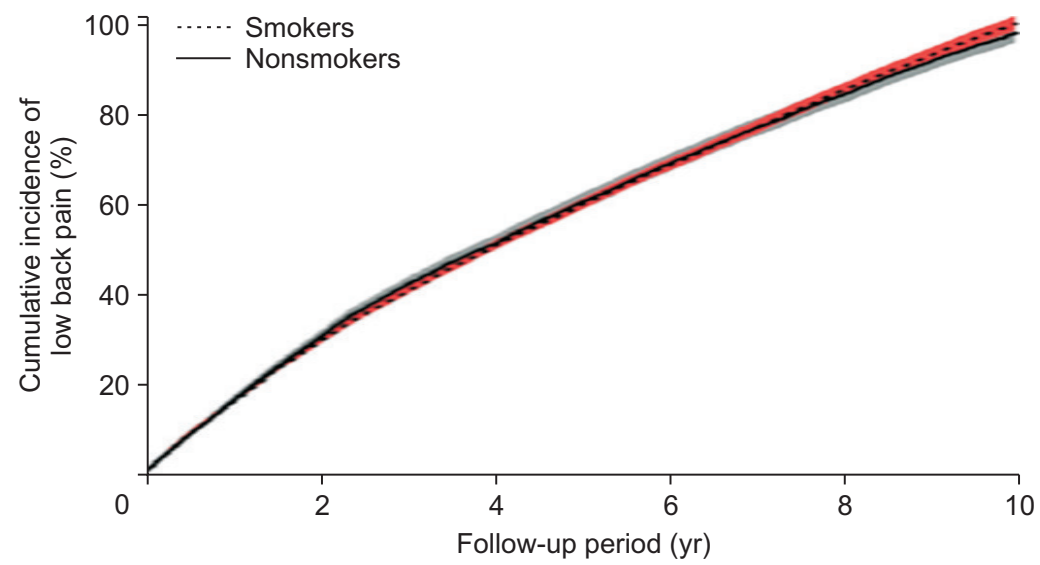

\begin{tabular}{|l|c|c|c|c|c|}
\hline No. at risk (nonsmokers) & & & & & \\
\hline Participants at risk & 3,038 & 2,064 & 1,763 & 1,532 & 1,367 \\
\hline Cumulative person-year & $8,846.99$ & $10,121.01$ & $12,267.03$ & $13,738.97$ & $14,957.93$ \\
\hline No. at risk (smokers) & & & & & \\
\hline Participants at risk & 10,411 & 7,421 & 6,300 & 5,881 & 5,270 \\
\hline Cumulative person-year & $30,066.93$ & $36,764.97$ & $43,964.51$ & $52,855.31$ & $57,760.89$ \\
\hline
\end{tabular}

Fig. 2. The cumulative incidence of low back pain by smoking exposure status in the male cohort $(204,066$ men older than 40 years) during the 10-year follow-up. The shading denotes $95 \%$ confidence intervals. The number of participants at risk and cumulative person-years are also shown.

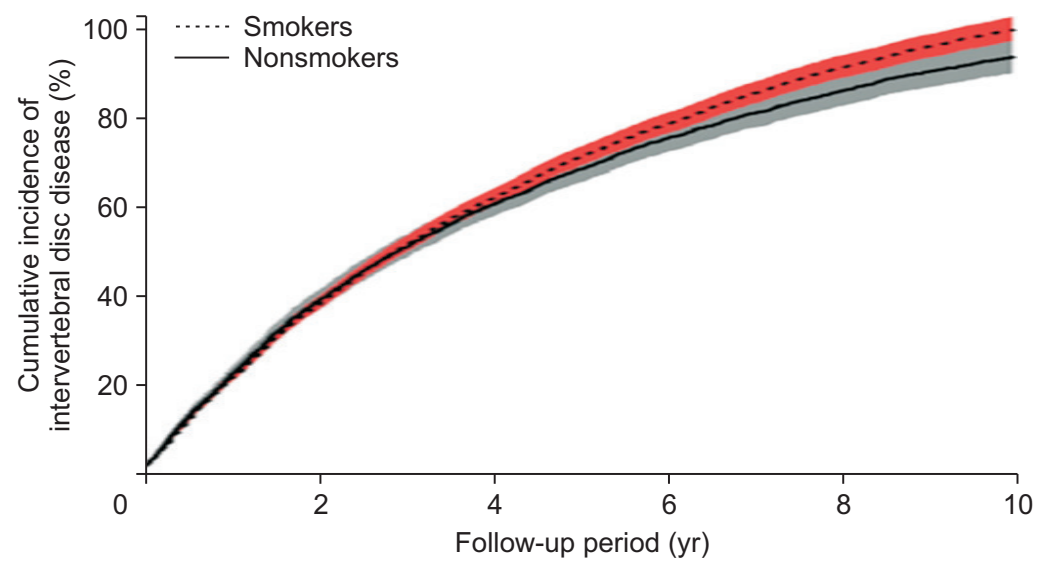

\begin{tabular}{|l|c|c|c|c|c|}
\hline No. at risk (nonsmokers) & & & & & \\
\hline Participants at risk & 1,777 & 963 & 666 & 475 & 342 \\
\hline Cumulative person-year & $4,998.88$ & $4,723.49$ & $4,627.8$ & $4,256.99$ & $3,732.99$ \\
\hline No. at risk (smokers) & & & & & \\
\hline Participants at risk & 6,177 & 3,781 & 2,671 & 2,024 & 1,361 \\
\hline Cumulative person-year & $17,508.98$ & $185,580.31$ & $18,494.04$ & $18,102.44$ & $14,885.3$ \\
\hline
\end{tabular}

Fig. 3. The cumulative incidence of intervertebral disc disease by smoking exposure status in the male cohort $(204,066$ men older than 40 years) during the 10-year follow-up. The shading denotes $95 \%$ confidence intervals. The number of participants at risk and cumulative person-years are also shown. 
Kwon et al. Prevalence of Spinal Disease among Smokers in Korea

Clinics in Orthopedic Surgery • Vol. 12, No. 2, $2020 \bullet$ www.ecios.org

In an unadjusted Cox model, the smoker group showed a higher cumulative incidence of LBP from 8 years after the start of follow-up than the nonsmoker group (Fig. 2). The smoker group also had a higher incidence of intervertebral disc disease from 4 years (Fig. 3), spinal stenosis from 5 years (Fig. 4), spinal instability from 1 year
(Fig. 5), or spondylolisthesis from 3 years (Fig. 6). In a multivariable-adjusted Cox model, the hazard ratio (HR) for LBP was higher in the smoker group (HR, 1.18; 95\% CI, 1.15 to 1.21), intervertebral disc disease (HR, 1.25; 95\% CI, 1.21 to 1.30 ), spinal stenosis (HR, 1.52; 95\% CI, 1.41 to 1.64 ), spinal instability (HR, 1.33; 95\% CI, 1.24 to 1.44 ),

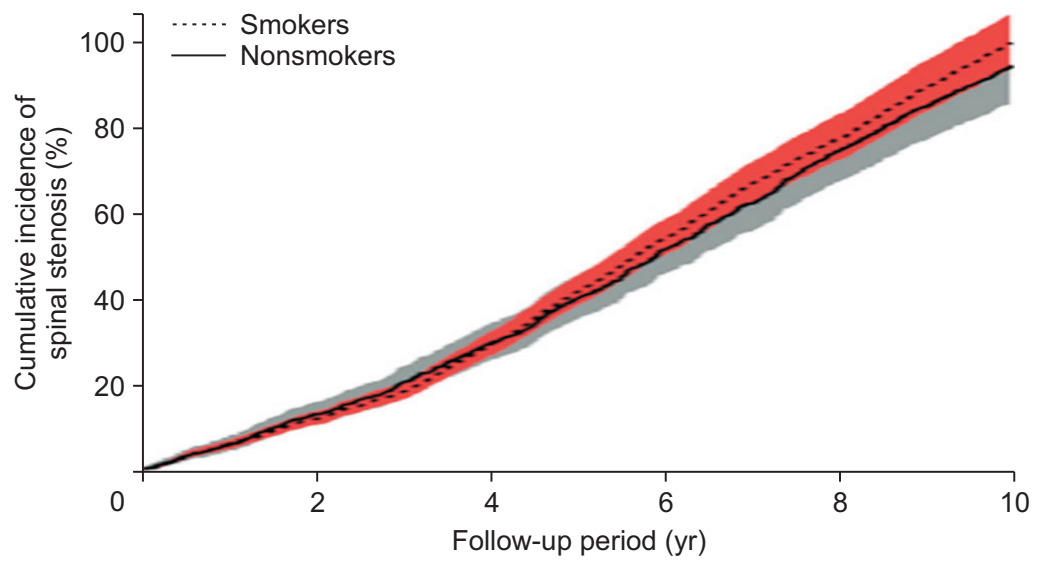

\begin{tabular}{|l|c|c|c|c|c|}
\hline No. at risk (nonsmokers) & & & & & \\
\hline Participants at risk & 132 & 167 & 215 & 228 & 196 \\
\hline Cumulative person-year & 387.53 & 847.01 & $1,518.69$ & $2,058.13$ & $2,152.59$ \\
\hline No. at risk (smokers) & & & & & \\
\hline Participants at risk & 432 & 600 & 862 & 809 & 778 \\
\hline Cumulative person-year & $1,261.61$ & $3,074.04$ & $6,031.04$ & $7,240.82$ & $8,532.97$ \\
\hline
\end{tabular}

Fig. 4. The cumulative incidence of spinal stenosis by smoking exposure status in the male cohort $(204,066$ men older than 40 years) during the 10 -year follow-up. The shading denotes $95 \%$ confidence intervals. The number of participants at risk and cumulative person-years are also shown.

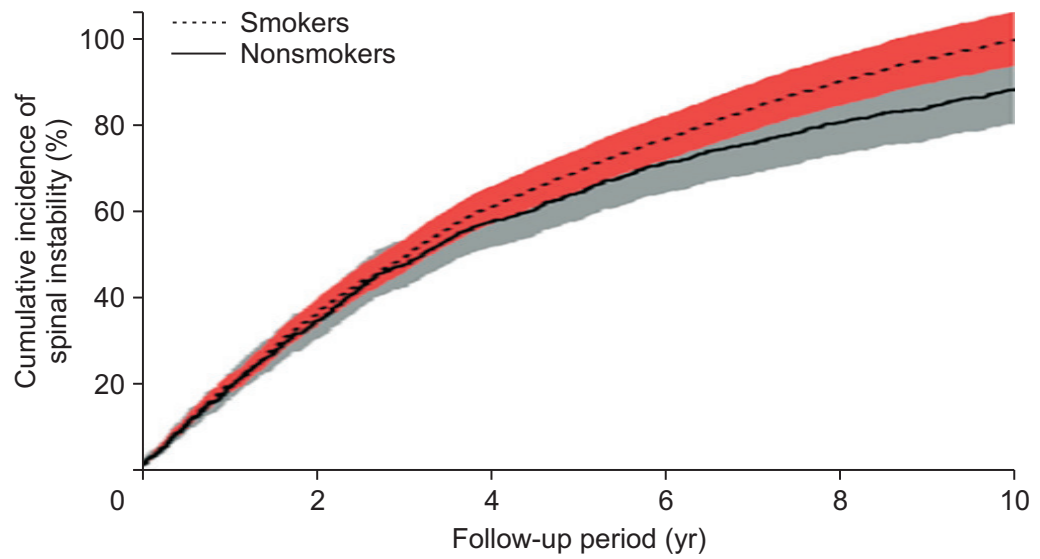

\begin{tabular}{|l|c|c|c|c|c|}
\hline No. at risk (nonsmokers) & & & & & \\
\hline Participants at risk & 363 & 237 & 140 & 98 & 79 \\
\hline Cumulative person-year & $1,041.89$ & $1,153.65$ & 978.10 & 880.80 & 871.98 \\
\hline No. at risk (smokers) & & & & & \\
\hline Participants at risk & 1,345 & 908 & 575 & 488 & 354 \\
\hline Cumulative person-year & $3,882.02$ & $4,470.05$ & $3,991.89$ & $4,358.17$ & $3,869.77$ \\
\hline
\end{tabular}

Fig. 5. The cumulative incidence of spinal instability by smoking exposure status in the male cohort $(204,066$ men older than 40 years) during the 10 -year followup. The shading denotes $95 \%$ confidence intervals. The number of participants at risk and cumulative person-years are also shown. 
Kwon et al. Prevalence of Spinal Disease among Smokers in Korea

Clinics in Orthopedic Surgery • Vol. 12, No. 2, $2020 \bullet$ www.ecios.org

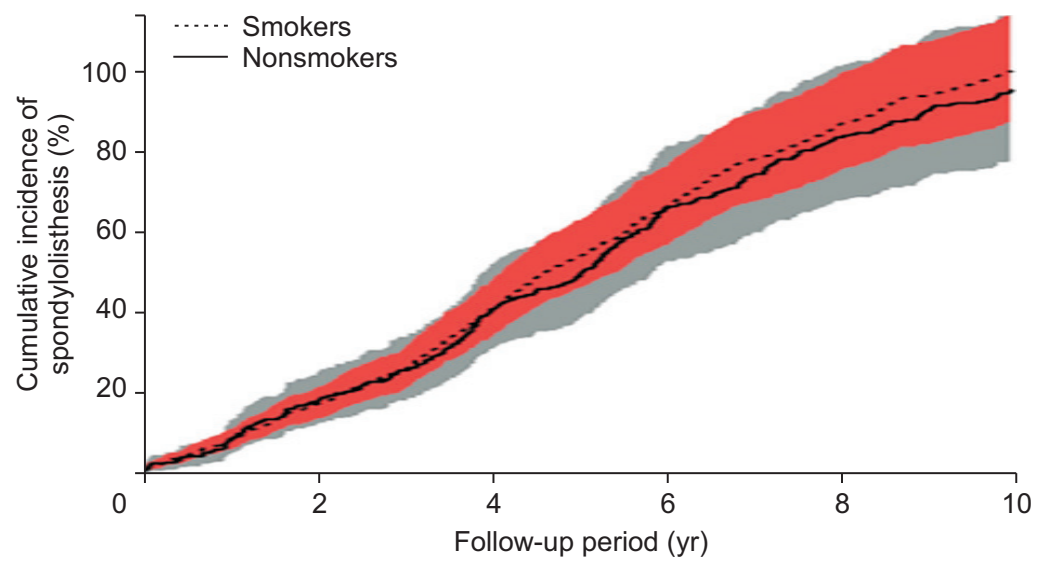

\begin{tabular}{|l|c|c|c|c|c|}
\hline No. at risk (nonsmokers) & & & & & \\
\hline Participants at risk & 45 & 57 & 66 & 46 & 30 \\
\hline Cumulative person-year & 134.87 & 296.94 & 470.48 & 416.57 & 330.85 \\
\hline No. at risk (smokers) & & & & & \\
\hline Participants at risk & 153 & 204 & 235 & 182 & 126 \\
\hline Cumulative person-year & 453.30 & $1,042.16$ & $1,628.16$ & $1,616.59$ & $1,374.80$ \\
\hline
\end{tabular}

Fig. 6. The cumulative incidence of spondylolisthesis by smoking exposure status in the male cohort $(204,066$ men older than 40 years) during the 10 -year followup. The shading denotes $95 \%$ confidence intervals. The number of participants at risk and cumulative person-years are also shown.

and spondylolisthesis (HR, 1.49; 95\% CI, 1.23 to 1.80 ) as compared with the nonsmoker group (Table 2).

\section{DISCUSSION}

In this retrospective cohort study of males aged 40 years and older, we found that the smoker group had a higher cumulative risk of developing LBP or lumbar spinal disorders such as intervertebral disc disease, spinal stenosis, spinal instability, and spondylolisthesis. The adjusted risk rate was the highest in individuals with spinal stenosis (52\%) and spondylolisthesis (49\%). To the best of our knowledge, this is the first study to investigate whether smokers are at higher risk than nonsmokers for four common back problems, such as intervertebral disc disease, spinal stenosis, spinal instability, and spondylolisthesis. In a recent metaanalysis of three cohort studies, smokers showed an overall $24 \%$ risk increase in LBP development; however, the sample size $(2,490$ in total) was smaller than that in this study, and these groups were not selected in the general population (occupational). ${ }^{5,13-15)}$ Unlike these studies, our study utilized a large, longitudinal, contemporary dataset of randomly selected national cohorts. Another strength of this study is that the size of the initial cohort $(n=514,866)$ was sufficient to select a well-balanced control group and the study design was appropriate to investigate the association between smoking and primary outcomes. To minimize the possibility of selection bias in retrospective cohort studies, we analyzed data after a 2-year clearance of patients with existing spinal disorders and back pain conditions. Furthermore, adjustments were made to anthropometric variables, clinical risk factors, and comorbidities. The use of lifestyle and socioeconomic data allowed adequate consideration of confounding factors. There are some limitations to this study. First, the retrospective observational design interfered with the evaluation of causality. Second, the reliability of specific information related to smoking was limited because it was based on self-reported data. Third, the analysis was confined to men because the percentage of female smokers (6\%) was too small to produce statistically meaningful results. Perhaps the reason for the low number of female smokers in this study is that female smokers are traditionally few in Korea and Korean women are reluctant to disclose their smoking status for social and cultural reasons. If the number of female smokers was sufficient to make a statistically meaningful analysis as in males, the effect of female smoking on the main outcome is not expected to be significantly different from male smoking. Fourth, the median follow-up period of the present study was 5.6 years, which is relatively longer than that in other studies. Still, it may not be enough time to investigate the incidence of LBP and related spinal diseases. A long-term follow-up of at least 10 or 20 years will clearly show the impact of smoking on the development of these diseases. Fifth, since participants of this study were mainly Asians, the results obtained from this study may not be general- 
Kwon et al. Prevalence of Spinal Disease among Smokers in Korea

Clinics in Orthopedic Surgery • Vol. 12, No. 2, $2020 \bullet$ www.ecios.org

ized to other populations.

In conclusion, smokers in male samples were at higher risk for LBP and related spinal diseases (intervertebral disc disease, spinal stenosis, spinal instability, and spondylolisthesis) than nonsmokers. Although the generalizability of our findings may be limited because of the observational study design and male-specific data, the results suggest that smoking can be a potential risk for LBP and related spinal diseases. Prospective cohort studies that involve at least 10 years of follow-up are needed to confirm our findings.

\section{CONFLICT OF INTEREST}

No potential conflict of interest relevant to this article was reported.

\section{ACKNOWLEDGEMENTS}

This work was supported by a grant from the National Health Insurance Service Ilsan Hospital (2015-20-007).

\section{REFERENCES}

1. GBD 2015 Disease and Injury Incidence and Prevalence Collaborators. Global, regional, and national incidence, prevalence, and years lived with disability for 310 diseases and injuries, 1990-2015: a systematic analysis for the Global Burden of Disease Study 2015. Lancet. 2016;388(10053):1545602.

2. Hoy D, March L, Brooks P, et al. Measuring the global burden of low back pain. Best Pract Res Clin Rheumatol. 2010; 24(2):155-65.

3. Carey TS, Garrett JM, Jackman A, Hadler N. Recurrence and care seeking after acute back pain: results of a long-term follow-up study: North Carolina Back Pain Project. Med Care. 1999;37(2):157-64.

4. Pengel LH, Herbert RD, Maher CG, Refshauge KM. Acute low back pain: systematic review of its prognosis. BMJ. 2003;327(7410):323.

5. Stanton TR, Henschke N, Maher CG, Refshauge KM, Latimer J, McAuley JH. After an episode of acute low back pain, recurrence is unpredictable and not as common as previously thought. Spine (Phila Pa 1976). 2008;33(26):2923-8.

6. Feldman DE, Shrier I, Rossignol M, Abenhaim L. Risk factors for the development of low back pain in adolescence. Am J Epidemiol. 2001;154(1):30-6.

7. Strine TW, Hootman JM. US national prevalence and correlates of low back and neck pain among adults. Arthritis Rheum. 2007;57(4):656-65.

8. Goldberg MS, Scott SC, Mayo NE. A review of the association between cigarette smoking and the development of nonspecific back pain and related outcomes. Spine (Phila $\mathrm{Pa}$
1976). 2000;25(8):995-1014.

9. Leboeuf-Yde C. Smoking and low back pain: a systematic literature review of 41 journal articles reporting 47 epidemiologic studies. Spine (Phila Pa 1976). 1999;24(14):146370 .

10. Shiri R, Karppinen J, Leino-Arjas P, Solovieva S, ViikariJuntura E. The association between smoking and low back pain: a meta-analysis. Am J Med. 2010;123(1):87.

11. Seong SC, Kim YY, Park SK, et al. Cohort profile: the National Health Insurance Service-National Health Screening Cohort (NHIS-HEALS) in Korea. BMJ Open. 2017;7(9): e016640.

12. Normand ST, Landrum MB, Guadagnoli E, et al. Validating recommendations for coronary angiography following acute myocardial infarction in the elderly: a matched analysis using propensity scores. J Clin Epidemiol. 2001;54(4):387-98.

13. Andersen JH, Haahr JP, Frost P. Risk factors for more severe regional musculoskeletal symptoms: a two-year prospective study of a general working population. Arthritis Rheum. 2007;56(4):1355-64.

14. Leino-Arjas P, Solovieva S, Kirjonen J, Reunanen A, Riihimaki H. Cardiovascular risk factors and low-back pain in a long-term follow-up of industrial employees. Scand J Work Environ Health. 2006;32(1):12-9.

15. Miranda H, Viikari-Juntura E, Punnett L, Riihimaki H. Occupational loading, health behavior and sleep disturbance as predictors of low-back pain. Scand J Work Environ Health. 2008;34(6):411-9. 